\title{
Author Correction: Retinal transcriptome and eQTL analyses identify genes associated with age-related macular degeneration
}

Rinki Ratnapriya, Olukayode A. Sosina, Margaret R. Starostik, Madeline Kwicklis, Rebecca J. Kapphahn, Lars G. Fritsche (D, Ashley Walton, Marios Arvanitis, Linn Gieser, Alexandra Pietraszkiewicz, Sandra R. Montezuma, Emily Y. Chew, Alexis Battle (D), Gonçalo R. Abecasis, Deborah A. Ferrington (D), Nilanjan Chatterjee (iD and Anand Swaroop (D)

Correction to: Nature Genetics https://doi.org/10.1038/s41588-019-0351-9, published online 11 February 2019.

In the version of this article initially published, in Supplementary Data 5, the logFC, FC, $P$ value and adjusted $P$ value for advanced AMD versus control (DE 4/1) without age correction did not correspond to the correct gene IDs. The errors have been corrected in the HTML version of the article.

Published online: 8 May 2019

https://doi.org/10.1038/s41588-019-0430-y

\section{Author Correction: New genetic signals for lung function highlight pathways and chronic obstructive pulmonary disease associations across multiple ancestries}

Nick Shrine (D), Anna L. Guyatt D, A. Mesut Erzurumluoglu, Victoria E. Jackson, Brian D. Hobbs (D), Carl A. Melbourne, Chiara Batini, Katherine A. Fawcett, Kijoung Song, Phuwanat Sakornsakolpat ID, Xingnan Li, Ruth Boxall, Nicola F. Reeve, Ma'en Obeidat D, Jing Hua Zhao, Matthias Wielscher, Understanding Society Scientific Group, Stefan Weiss $\mathbb{D}_{\text {, }}$ Katherine A. Kentistou (D), James P. Cook, Benjamin B. Sun D, Jian Zhou, Jennie Hui, Stefan Karrasch, Medea Imboden, Sarah E Harris, Jonathan Marten, Stefan Enroth (D), Shona M. Kerr, Ida Surakka, Veronique Vitart (D), Terho Lehtimäki, Richard J. Allen (D), Per S. Bakke, Terri H. Beaty DiD, Eugene R. Bleecker, Yohan Bossé D , Corry-Anke Brandsma, Zhengming Chen, James D. Crapo, John Danesh, Dawn L. DeMeo, Frank Dudbridge, Ralf Ewert, Christian Gieger, Amund Gulsvik, Anna L. Hansell, Ke Hao, Joshua D. Hoffman, John E. Hokanson, Georg Homuth, Peter K. Joshi (D), Philippe Joubert, Claudia Langenberg, Xuan Li, Liming Li, Kuang Lin, Lars Lind, Nicholas Locantore, Jian'an Luan, Anubha Mahajan (D), Joseph C. Maranville, Alison Murray D, David C. Nickle, Richard Packer, Margaret M. Parker, Megan L. Paynton, David J. Porteous (D), Dmitry Prokopenko, Dandi Qiao, Rajesh Rawal, Heiko Runz, Ian Sayers (D), Don D Sin, Blair H Smith (D), María Soler Artigas, David Sparrow, Ruth Tal-Singer (D), Paul R. H. J. Timmers (D), Maarten Van den Berge, John C. Whittaker, Prescott G. Woodruff, Laura M. Yerges-Armstrong, Olga G. Troyanskaya D, Olli T. Raitakari, Mika Kähönen, Ozren Polašek, Ulf Gyllensten, Igor Rudan (D, Ian J. Deary, Nicole M. Probst-Hensch, Holger Schulz, Alan L James, James F. Wilson (D), Beate Stubbe, Eleftheria Zeggini (D), Marjo-Riitta Jarvelin, Nick Wareham, Edwin K. Silverman, Caroline Hayward (D), Andrew P. Morris (D), Adam S. Butterworth (D), Robert A. Scott, Robin G. Walters (D), Deborah A. Meyers, Michael H. Cho (D), David P. Strachan (D), Ian P. Hall, Martin D. Tobin (D) and Louise V. Wain (D)

Correction to: Nature Genetics https://doi.org/10.1038/s41588-018-0321-7, published online 25 February 2019.

In the version of this article initially published, the $y$-axis labels in Fig. 5 read 'signed $\log _{10}[\mathrm{FDR}]$ ' but should have read '- $\log _{10}[\mathrm{FDR}]$ ', and the figure legend incorrectly indicated that directions of effect are shown in Fig. 5 rather than Supplementary Table 25. The errors have been corrected in the HTML and PDF versions of the article. 